\title{
A low-noise preamplifier for $\gamma$-ray sensors with add-on device for large-signal management
}

\author{
Alberto Pullia*, Francesca Zocca \\ Department of Physics and INFN, University of Milano, via Celoria 16, I-20133 Milano, Italy
}

Received 8 September 2004; received in revised form 18 January 2005; accepted 5 February 2005

Available online 19 April 2005

\begin{abstract}
A new charge-sensitive preamplifier for High Purity Germanium $\gamma$-ray detectors has been built and tested. It comprises an over-load recovery device that reduces the dead time brought about by hits of energetic particles. The amplitude of the large signals is reconstructed from Time-Over-Threshold measurements performed along the reset transients. Using such a technology an extremely wide energy range of $10 \mathrm{keV}$ to $30 \mathrm{MeV}$, or $70 \mathrm{~dB}$, is obtained. With a count rate of $6 \mathrm{kHz}$ a resolution of $1.1 \mathrm{keV}$ fwhm is obtained on the ${ }^{152} \mathrm{Eu} 122 \mathrm{keV}$ line and of $\sim 5$ perthousand on pulser lines in the energy range of $5-30 \mathrm{MeV}$.
\end{abstract}

(C) 2005 Elsevier B.V. All rights reserved.

PACS: 84.30.-r; 29.30.Kv

Keywords: Low noise preamplifier; Wide dynamic range preamplifier; Fast reset preamplifier; $\gamma$-ray spectroscopy

\section{Introduction}

The typical energy range of state-of-the-art $\gamma$-ray spectrometers employing High purity Germanium (HPGe) detectors is from a few $\mathrm{keV}$ to a few $\mathrm{MeV}$. Hits of photons/particles of larger energies can easily put the preamplifier or the pulse-height analyzer into saturation and introduce a significant system dead time. We suggest

\footnotetext{
*Corresponding author. Tel.: +39250317735; fax: +39250317487 .

E-mail address: alberto.pullia@mi.infn.it (A. Pullia).
}

that a new time-variant preamplifier can address these issues reducing the dead time and extending the accepted energy range by one order of magnitude. In the typical amplitude range it behaves like a classic charge-sensitive preamplifier for HPGe $\gamma$-ray sensors, with a resistive continuous reset of the detector charge stored on the feedback capacitance. For large signals, however, a switchable device turns on which swiftly desaturates the preamplifier. This device plays the role of a large-signal manager and helps reduce the dead time brought about by energetic events with little or no sacrifice of signal-to-noise ratio in 
the typical energy range. Furthermore, a relationship exists between the width of the desaturation transient and the amplitude of the large signal. It is so possible to accurately estimate the large-signal amplitude from a Time Over Threshold (TOT) measurement [1,2] performed along the desaturation transient. As a result the amplitude range of the preamplifier is substantially increased. Using this technology we were able to measure signals in the range $10 \mathrm{keV}$ to $30 \mathrm{MeV}$, as compared to the typical $10 \mathrm{keV}$-to- $2 \mathrm{MeV}$ range of most low-noise preamplifiers for $\gamma$-ray spectroscopy. Such a wide energy range is of interest for the last generation of nuclear-physics experiments [3,4] with bulky $4 \pi$ germanium detectors. In fact, as shown by Montecarlo simulations, a $9 \mathrm{~cm}$ Ge layer still has a detection efficiency of 9 to $14 \%$ for $\gamma$ rays of 20 to $30 \mathrm{MeV}$ [5]. Note that the first-interaction mechanism at these energies is pair production. In the paper the new preamplifier as well as the TOT algorithm used to measure the large signals will be discussed. Experimental results will be shown.

\section{Circuit architecture}

In the foregoing discussion we assume that the polarity of the detector current signals is negative (anodic signals). The architecture of the proposed time-variant preamplifier is shown in Fig. 1. It comprises a charge sensitive stage ("integration stage"), a $\mathrm{P} / \mathrm{Z}$ amplifier, a differential output buffer, and an add-on Large-Signal Manager (LSM). The LSM consists of an inverting Schmitt-trigger comparator and a temperature stabilized switchable current sink. The LSM continuously senses the amplitude of the detector signals. When signals of normal amplitude occur the LSM keeps the current sink in the off condition. When a large signal arrives it switches the current sink on, which absorbs charge from capacitance $\mathrm{C}$ of the $\mathrm{P} / \mathrm{Z}$ stage until the preamplifier output voltage is brought back to its quiescent value. At this point the comparator automatically turns over and switches the current sink off so completing the fast-reset procedure. The preamplifier is then ready to process new detector signals.

It must be observed that the reset procedure does not remove the physical charge delivered by the detector and stored on the integration capacitance $C_{\mathrm{F}}$. This has advantages and disadvantages. The advantage is that no wire is connected to the preamplifier input, i.e. the most sensitive and critical point of the front-end electronics. This means that no electrical parasitics (capacitance, current, lossy dielectrics) are added at the pre-

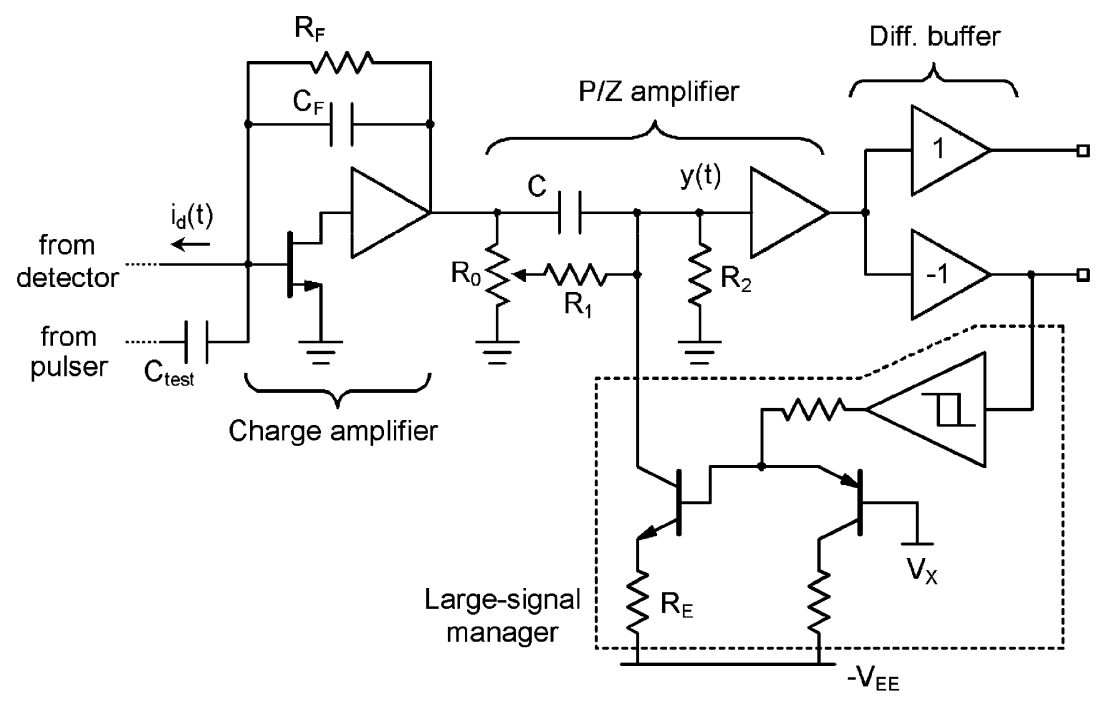

Fig. 1. Architecture of the time-variant preamplifier. 
amplifier input, which greatly helps keeping the electronic noise low. The disadvantage is that the integration stage itself is not protected against saturation by the large-signal manager. Luckily, saturation of the integration stage is a relatively rare event. In fact, assuming $C_{\mathrm{F}}=1 \mathrm{pF}$, the gain of the integration stage is relatively low, or $55 \mathrm{mV} /$ $\mathrm{MeV}$ for a $\mathrm{Ge}$ detector, which means that an individual $100 \mathrm{MeV}$ event yields a signal swing of only $5.4 \mathrm{~V}$. Using $12 \mathrm{~V}$ power supply this is well below the saturation point of the integration stage. If a random sequence of energetic events occurs, the mean value of the waveform seen at the integration stage output could shift if the detector is DC coupled to the preamplifier. We can calculate such a shift $\Delta \mathrm{V}$ as the voltage drop caused by the mean signal current through resistor $R_{\mathrm{F}}$ or

$\Delta V=(q E / \psi) v R_{\mathrm{F}}$

where $\psi=2.92 \mathrm{eV} /$ pair is the mean energy necessary to create an electron-hole pair in germanium, $q$ is the electron charge, $E$ is the mean energy release, and $v$ is the event rate. As an example assuming $E=30 \mathrm{MeV}, R_{\mathrm{F}}=1 \mathrm{G} \Omega$ and $v=6 \mathrm{kHz}$ we obtain $\Delta V \approx 10 \mathrm{~V}$, which is still acceptable.

If instead the detector is AC coupled to the preamplifier, the mean signal current will flow through the resistor used to supply the High Voltage to the detector electrode, causing a voltage drop on the electrode itself. However this voltage would be blocked by the AC coupling capacitor. So, this latter case poses no concerns about DC shifts of the output waveform at increasing event rates.

\section{Fast-reset transient}

We are now to calculate the reset-transient shape as well as its width as seen at the PZ network output. To do so we assume that a large signal occurs upon a tail of one or more regular signals. Fig. 2a shows a positive such signal as seen at the $\mathrm{P} / \mathrm{Z}$ network output. Fig. $2 \mathrm{~b}$ shows the corresponding signal seen at the Schmitt-trigger comparator output.

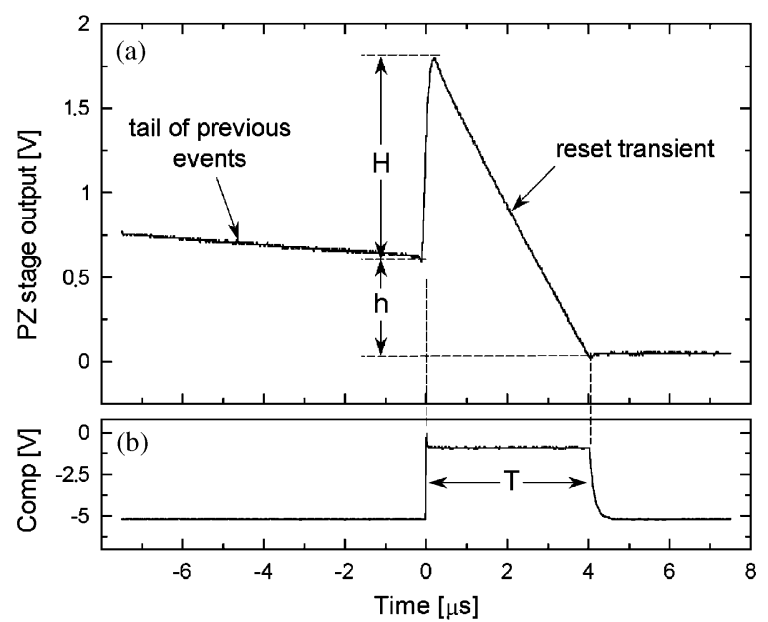

Fig. 2. (a) Large signal $(H)$ upon a tail $(h)$ at the PZ stage output. (b) Schmitt-trigger comparator signal.

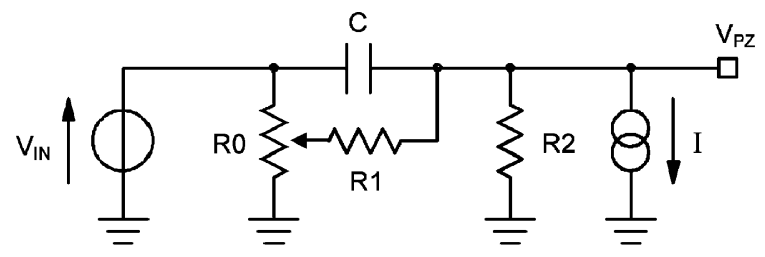

Fig. 3. Reset current through $\mathrm{P} / \mathrm{Z}$ network.

Along the reset transient the current sink of the LSM is in the on condition, and continuously absorbs charge from capacitance $C$ of the $\mathrm{P} / \mathrm{Z}$ network. This condition is depicted in Fig. 3, where

$V_{\text {IN }}=(h+H) \mathrm{e}^{-\frac{t}{\tau_{F}}}$

is the exponential tail of the previous event seen at the integration stage output, $\tau_{\mathrm{F}}=R_{\mathrm{F}} C_{\mathrm{F}}$ is the pole of the integration stage, and

$I=\left(V_{\mathrm{EE}}-V_{\mathrm{X}}\right) / R_{\mathrm{E}}$

is a constant current. $V_{\mathrm{EE}}, V_{\mathrm{X}}$ and $R_{\mathrm{E}}$ are the symbols shown in Fig. 1. Parameters $H$ and $h$ shown in Fig. 2 are, respectively, the height of the large signal and that of the tail.

Assuming $R_{0} \ll R_{1}$, and after trimming $R_{0}$ in such a way that the zero of the $\mathrm{P} / \mathrm{Z}$ network cancels the pole of the integration stage it turns out, after a bit of calculations 
(see Appendix), that

$V_{\mathrm{PZ}}=\left(h+H+I R_{1} \oplus R_{2}\right) \mathrm{e}^{-\frac{t}{\tau_{P}}}-I R_{1} \oplus R_{2}$

where symbol " $\oplus$ " stands for "parallel connection of" and $\tau_{P}=C R_{1} \oplus R_{2}$ is the pole of the $\mathrm{P} / \mathrm{Z}$ network. By equating Eq. (4) to zero and solving for $t$, we derive the reset time $T$, or

$T=\tau_{P} \log \left(1+\frac{H+h}{I R_{1} \oplus R_{2}}\right)$.

We can now rearrange Eq. (5) in the form

$H=I R_{1} \oplus R_{2}\left(\mathrm{e}^{\frac{T}{\mathrm{e}_{P}}}-1\right)-h$

which yields the height of the large signal as a function of the reset time $T$ and the tail $h$. The tail $h$ is obtained in practice as the difference of the pre- and post-pulse baselines. Averaging along these baseline spans helps keep the noise low. It is useful to expand the exponential term of (6), which yields

$H=\frac{I}{C} T+\frac{I}{2 C \tau_{P}} T^{2}+\ldots-h$.

Eq. (7) can be further simplified if the tail is negligible $(h \rightarrow 0)$ and the reset time $T$ is substantially shorter than $\tau_{P}$. In this case the second- and higher-order terms of $T$ can be neglected. With these assumptions Eq. (7) yields

$H \approx \frac{I}{C} T$

which establishes a linear relation between the reset time and the amplitude of the large signal. This relation is rather intuitive. In fact (8) corresponds to the voltage drop caused by a constant current $I$ flowing through capacitor $C$ of the PZ stage for a time $T$. The presence of higherorder terms depends on the fact that current $I$ not only flows through $C$, but also through resistors $R_{1}$ and $R_{2}$, as shown in Fig. 3, which yields a nonlinear dependence of $H+h$ on $T$. It is worth giving a simple geometrical interpretation of (8): $T$ is the horizontal edge of a right triangle and $H$ its vertical edge. The former can be projected to the latter by multiplying it, i.e. $T$, by the slope of the hypotenuse, i.e. $I / C$.

It is now useful to show the sensitivity seen at the PZ stage output as obtained from obvious calculations,

$\frac{V_{\mathrm{PZ}}}{E}=\frac{q}{\psi} \frac{1}{C_{\mathrm{F}}}$

where $E$ is the event-energy and $V_{\mathrm{PZ}}$ is the voltage swing observed at the PZ stage output (see Fig. 3). By dividing Eq. (7) by the gain (9), $H$ and $h$ get expressed in terms of the energy of the large signal $\left(E_{w}\right)$ and that of the tail $\left(E_{c}\right)$, which yields

$E_{w}=\psi \frac{I}{q} \frac{C_{\mathrm{F}}}{C} T+\psi \frac{I}{2 q} \frac{C_{\mathrm{F}}}{C \tau_{P}} T^{2}+\ldots-E_{c}$

and, by dividing (8) by (9),

$E_{w} \approx \psi \frac{I}{q} \frac{C_{\mathrm{F}}}{C} T$.

Eqs. (10) and (11) permit to reconstruct the largesignal energy from the reset time and the tail. It is worth observing that Eq. (11) yields

$\frac{E_{w}}{T} \approx \psi \frac{I}{q} \frac{C_{\mathrm{F}}}{C}$.

Using (12) the fast-reset velocity can be derived from a few circuit parameters. For example with $C_{\mathrm{F}}=1 \mathrm{pF}, C=4.7 \mathrm{nF}, I=2 \mathrm{~mA}$ one obtains a reset velocity of $7.8 \mathrm{MeV} / \mu \mathrm{s}$, which means that a $100 \mathrm{MeV}$ signal is fully reset in about $13 \mu \mathrm{s}$.

\section{Experimental measurements}

In the experimental part of this work we used a custom-made preamplifier equipped with the addon LSM device sketched in Fig. 1, connected to a state-of-the-art closed-end cylindrical HPGe detector. To implement the Schmitt trigger of the LSM we used a fast comparator mod. LT1719 from Linear Technologies with a resistive positive feedback arranged in such a way to get $V_{\mathrm{T} 1}=0 \mathrm{~V}$ and $V_{\mathrm{T} 2}=1.1 \mathrm{~V}$ as switch-over thresholds. A photograph of the preamplifier is shown in Fig. 4. We used ${ }^{152} \mathrm{Eu}$ and ${ }^{60} \mathrm{Co}$ calibration sources placed at different distances from the detector, obtaining overall counting rates in the range $1-32 \mathrm{kHz}$. We could also artificially simulate energetic events in the range $5-27 \mathrm{MeV}$ by providing large signals at the preamplifier input with a spectroscopy-grade pulser and a calibrated test 


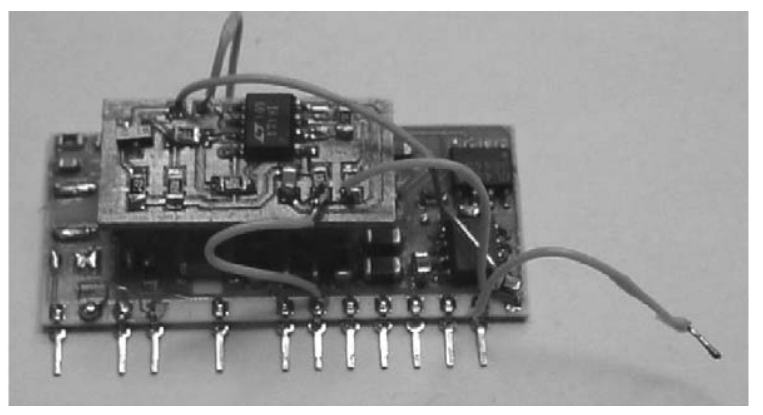

Fig. 4. Photograph of the used prototype preamplifier.

capacitance. The pulser signals were large enough to trigger the LSM device. The fast-reset transients were superposed to the tails caused by the distribution of $\gamma$ photons delivered by the mentioned radioactive sources. We collected a complete dataset with an 8-bit $1 \mathrm{GSa} / \mathrm{s}$ digital oscilloscope linked to a host PC, recording the waveforms seen at the preamplifier analog output as well as those provided by the Schmitt-trigger comparator.

\subsection{Preliminary calibrations}

First of all we needed a calibration of the test capacitor $C_{\text {test }}$. For this purpose we acquired a full spectrum of ${ }^{152} \mathrm{Eu}$ using a quasi-Gaussian spectroscopy amplifier and a Pulse-Height Analyzer (PHA) and we grew a host of pulser lines in the low- and high-energy regions of the acquired $\gamma$ photon spectrum. We could hence estimate the injected charge as well as $C_{\text {test }}$ looking at the position of the pulser lines along the $x$-axis. The test capacitance value turned out to be $(0.222 \pm 0.027) \mathrm{pF}$.

Then, from the acquired spectrum we calibrated the energy-to-voltage gain of the preamplifier, which turned out to be $G=(541.86 \pm 0.65) \mathrm{mV} / \mathrm{MeV}$. Because the differential buffer has a gain of $\sim 3$ the feedback capacitance $C_{\mathrm{F}}$ turned out to be $\sim 0.3 \mathrm{pF}$.

Because the test and the feedback capacitance were mounted in the cryostat in close proximity to the detector readout electrode, we had no possibility to change/optimize their value. Note that a low value of $C_{\text {test }}$ makes it difficult to inject large pulser signals. For example, in order to inject a charge of $1650 \mathrm{fC}$, corresponding to an energy of $30 \mathrm{MeV}$, a pulser signal as large as $4.72 \mathrm{~V}$ should be provided. The low value of $C_{\mathrm{F}}$, instead, slows down the reset mechanism, as shown by Eq. (12).

\subsection{Algorithm for large-signal reconstruction}

In this paragraph we describe in detail all steps necessary in practice to reconstruct the energy of the large signals. We assume that the large signals are superposed to a distribution of low-energy signals, whose tails play the role of a stochastic noise in this case. To perform the measurement of a large signal we need both the waveform provided by the preamplifier and that of the Schmitt-trigger comparator. Whenever a large signal occurs the comparator switches over and delivers a rectangular pulse of width $T$. The switch over times $t_{1}$ and $t_{2}$ yield the start and stop points of the fast reset transient as well as the width $T$ of the comparator pulse, i.e. $T=t_{2}-t_{1}$. To better define $t_{1}$ and $t_{2}$ we set both logical thresholds at one half of the comparator voltage swing. If the comparator signal is converted into a digital form with an ADC, accurate estimates of $t_{1}$ and $t_{2}$ can be made numerically with interpolation algorithms. After measuring $T$ and using Eq. (10) we can estimate the energy $E_{w}$ of the large signal as

$E_{w}=b_{1} T+b_{2} T^{2}-E_{c}$

where $b_{1}$ and $b_{2}$ are parameters not depending on $T$, and $E_{c}$, i.e. the tail, is

$E_{c}=E_{1}-E_{2}+E_{0}$

where $E_{1}$ and $E_{2}$ are the averaged pre- and postpulse baseline estimates expressed in energy and $E_{0}$ is an offset term. It is worth pointing out that $E_{w}$ and $E_{c}$ correspond exactly to $H$ and $h$ of Fig. 2 where voltage is converted into energy. Expressing now $E_{1}-E_{2}$ in terms of the baseline voltages $V_{1}$ and $V_{2}$, Eq. (14) becomes

$E_{c}=\left(V_{1}-V_{2}\right) / G+E_{0}$

where $G$ is the energy-to-voltage gain derived in the preliminary calibrations. Substituting Eq. (15) 
into Eq. (13) we finally obtain

$E_{w}=b_{1} T+b_{2} T^{2}-\left(V_{1}-V_{2}\right) / G-E_{0}$

which gives the large-signal energy as a function of the reset time $T$ and the pre- and post-pulse voltage baselines.

\subsection{Parameter estimation}

An estimate of $G$ is available from the preliminary calibration procedure. We need an estimate for $b_{1}, b_{2}$, and $E_{0}$. By comparing (16) with (10) one obtains the theoretical relations

$b_{1}=\psi \frac{I}{q} \frac{C_{\mathrm{F}}}{C}$

$b_{2}=\frac{b_{1}}{2 \tau_{P}}$

Eqs. (17) and (18) however cannot be used reliably because $C_{\mathrm{F}}$ and $C$ are not calibrated. Parameters $b_{1}, b_{2}$, as well as $E_{0}$, however, can be estimated with a different approach. This consists of providing a host of large pulser signals, the energy of which is known from energy-to-voltage calibration, or

$E_{w}=(\psi / q) C_{\text {test }} V_{\mathrm{p}}$

where $V_{\mathrm{p}}$ is the voltage swing of the pulser signals. Substituting Eq. (19) into Eq. (16) yields

$(\psi / q) C_{\text {test }} V_{p}+\left(V_{1}-V_{2}\right) / G=b_{1} T+b_{2} T^{2}-E_{0}$.

Eq. (20) establishes a linear relation between measured data and parameters $b_{1}, b_{2}$, and $E_{0}$. These latter parameters can hence be derived with a simple least-mean-squares (LMS) algorithm, provided that three or more experimental datasets are available to evaluate Eq. (20), each having a different value of $T$. In practice we repeatedly supplied pulser signals of four amplitudes in the energy range from 5 to $27 \mathrm{MeV}$. We had no possibility to inject larger signals because of the low value of $C_{\text {test }}$ and the limited voltage range of the pulser. To generate a significant distribution of disturbing tails we contextually irradiated the detector with an ${ }^{152} \mathrm{Eu}$ source placed in such a way to get an event rate of $2 \mathrm{kHz}$. We then used
Table 1

Parameter estimates

\begin{tabular}{lll}
\hline$b_{1}$ & $b_{2}$ & $E_{0}$ \\
\hline $3.5891 \mathrm{MeV} / \mu \mathrm{s}$ & $0.02144 \mathrm{MeV} / \mu \mathrm{s}^{2}$ & $1.5590 \mathrm{MeV}$ \\
\hline
\end{tabular}

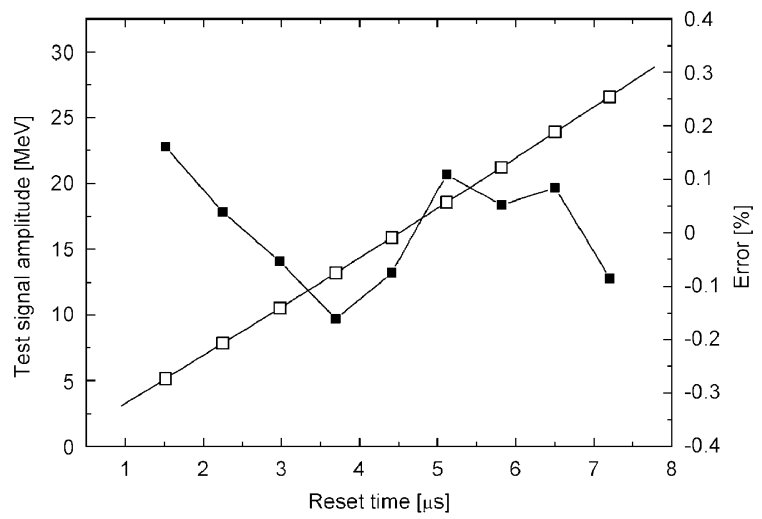

Fig. 5. Parabolic fitting of the wide-signal amplitude vs. the reset time $T$ (curve through the open squares, left $y$ axis). The error is shown with solid squares (right $y$ axis).

2000 equations of the type (20), as obtained from such dataset, and extracted the parameters with a LMS procedure. The so-obtained estimates of $b_{1}$, $b_{2}$, and $E_{0}$ are shown in Table 1 . The mean slope of the reset transients was $3.6 \mathrm{MeV} / \mu$ s. Of course this slope could be increased, if needed, by increasing the reset current $I$.

Some of the measured values of $E_{w}$ vs. $T$ are shown in Fig. 5 in open squares. In this case we reduced the event rate to have few significant tails. The parabolic fitting is shown in solid line. The error (solid squares) is of the order of $\pm 0.1 \%$ in the shown range. Using the calibrated parameters of Table 1 we were eventually ready to estimate the large-signal energy of any event from Eq. (16).

\subsection{Data acquisitions}

In order to test the large-signal measurement technique, we irradiated the detector with a ${ }^{60} \mathrm{Co}$ source placed in such a way to get an event rate of $32 \mathrm{kHz}$, and injected large pulser signals of $10 \mathrm{MeV}$ 


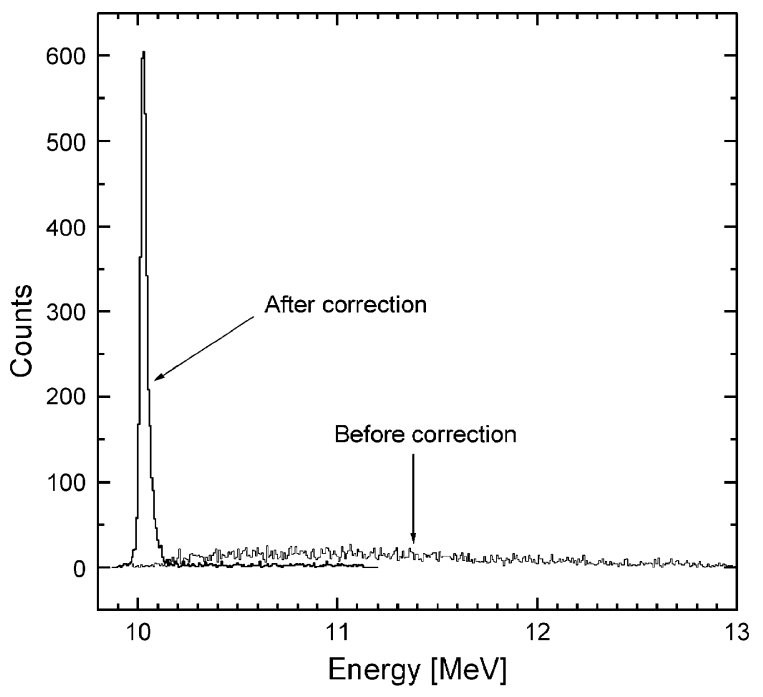

Fig. 6. Pulser line. A background of 32 kcounts/s of ${ }^{60} \mathrm{Co}$ signals is present and destroys the large-signal resolution if no correction is made.

at a rate of a few $\mathrm{Hz}$. We then histogrammed the large-signal energy as reconstructed with tail correction from Eq. (16). As a comparison we also histogrammed the large signals as reconstructed with no tail correction, i.e. from Eq. (11). The results are shown in Fig. 6. The effectiveness of tail correction is evident: when no correction is made the line completely disappears as the stochastic distribution of tails scrambles it out. When the correction is made the line gets nicely reconstructed showing a relative fwhm as good as $\sim 6$ per thousand, or $56 \mathrm{keV} @ \sim 10 \mathrm{MeV}$.

We then irradiated the detector with a ${ }^{152} \mathrm{Eu}$ source placed in such a way to get an event rate of $6 \mathrm{kHz}$, and injected large pulser signals in the energy range from 5 to $27 \mathrm{MeV}$. We histogrammed both the low-energy signals of the ${ }^{152} \mathrm{Eu}$ source, using a PHA and a quasi-Gaussian shaper set at $3 \mu$ s of shaping time, and the large signals, using a digital oscilloscope, a personal computer and a numerical filter. To reconstruct the large signals we recorded both the preamplifier and the comparator signals, and implemented Eq. (16) evaluating the pre- and post-pulse baseline by averaging, respectively, along 0.2 and $1.4 \mu$ s time spans, as shown in Fig. 7. As a result we got the overall

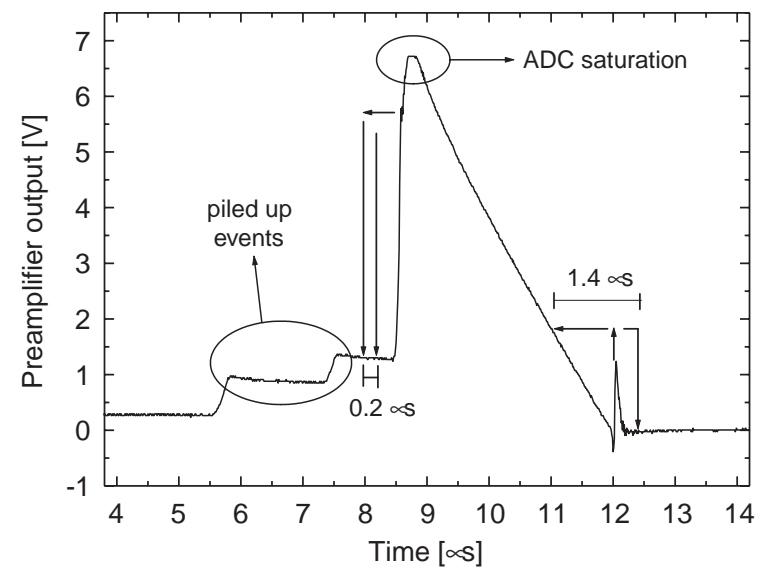

Fig. 7. Positions of time spans $T_{1}$ and $T_{2}(0.2$ and $1.4 \mu \mathrm{s})$ used to evaluate the pre- and post-pulse baselines. Note that $T_{2}$ is partially overlapped to the final part of the reset curve. This is useful to avoid stochastic errors due to events in the close postpulse region. This also yields a deterministic offset in the estimate of the post-pulse baseline, which, however, is taken into account by the model through term $E_{0}$. The narrow spike is due to cross-talk from the comparator and also contributes to $E_{0}$.

spectrum shown in Fig. 8 in the extremely wide range from $10 \mathrm{keV}$ to $27 \mathrm{MeV}$.

All ${ }^{152} \mathrm{Eu}$ lines are well resolved, the $122 \mathrm{keV}$ line having a fwhm of $\approx 1.1 \mathrm{keV}$. The high-energy lines are also finely resolved, having a relative fwhm of the order of 5 perthousand. The dependence of the large-signal resolution on the energy and on the event rate is shown in Figs. 9a and b. In both figures two cases are shown, in which the comparator signal is sampled with the oscilloscope 8-bit ADC or with a single-bit ADC, i.e. with a simple high/low codification. The case for an 8-bit ADC yields a higher resolution because in this case a sampled waveform is available and the switch over times, and hence the reset time, can be derived with a higher precision by numeric interpolation algorithms.

Figs. 8 and 9a show that the resolution of the reconstructed large signal worsens as the signal amplitude is increased. An explanation of this effect is that the larger the signal the longer the reset time, and therefore the larger the probability that a low-energy signal occurs along the reset transient. The presence of such 


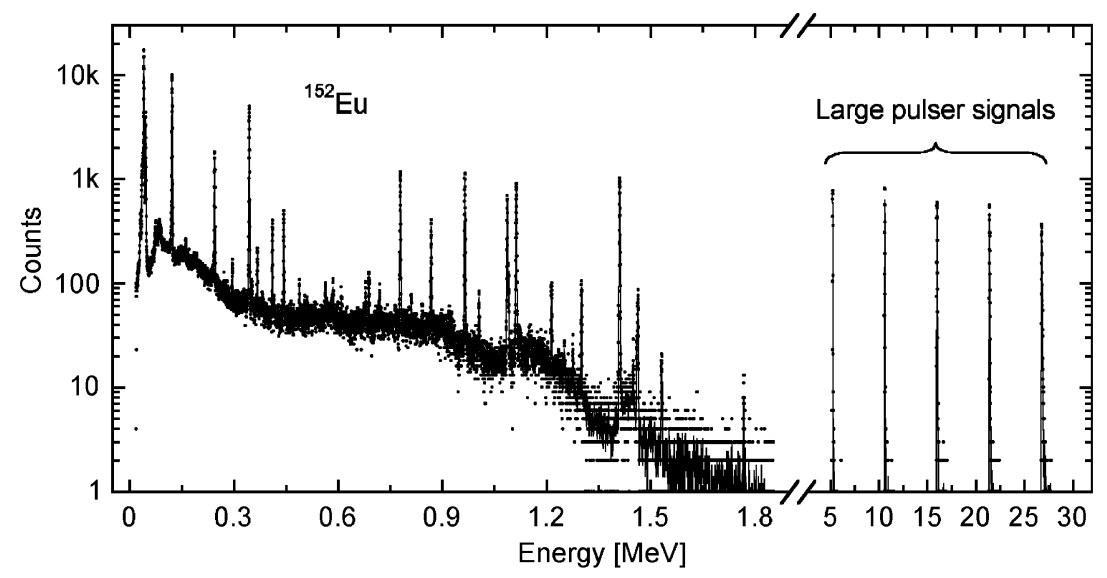

Fig. 8. Spectrum of ${ }^{152} \mathrm{Eu}$ with high-energy pulser lines. The average event rate is $6 \mathrm{kHz}$.
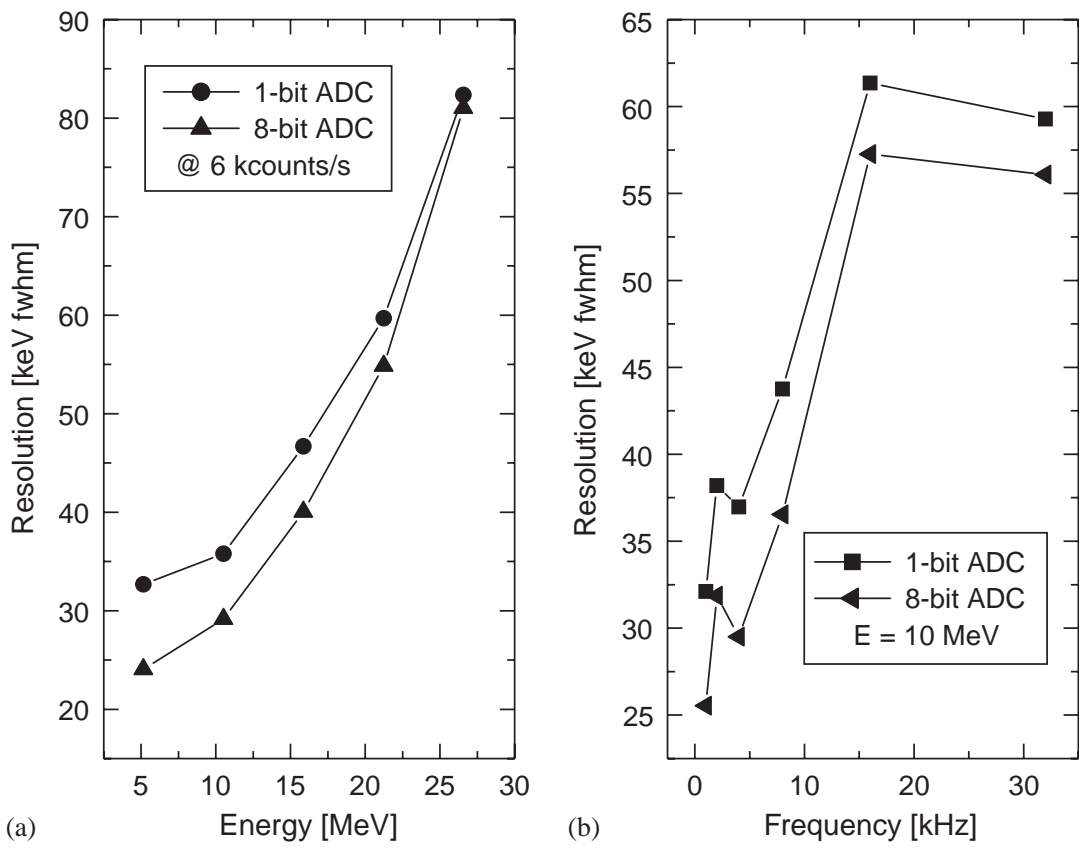

Fig. 9. Resolution of the pulser lines as a function of (a) the signal energy or (b) the event rate.

piled up events acts as an additive stochastic noise. Fig. 10 shows an example of a piled up event along the reset transient. It can be seen that it yields an increased reset time and, inherently, an error in the reconstructed largesignal energy.

\section{Appendix}

We can find $V_{\mathrm{PZ}}$ using the superposition theorem as the sum of the signals $V_{\mathrm{PZ} 1}$ and $V_{\mathrm{PZ} 2}$ obtained by switching on separately inputs $V_{\mathrm{IN}}$ and $I$. Let us begin calculate $V_{\mathrm{PZ1}}$. Assuming that 


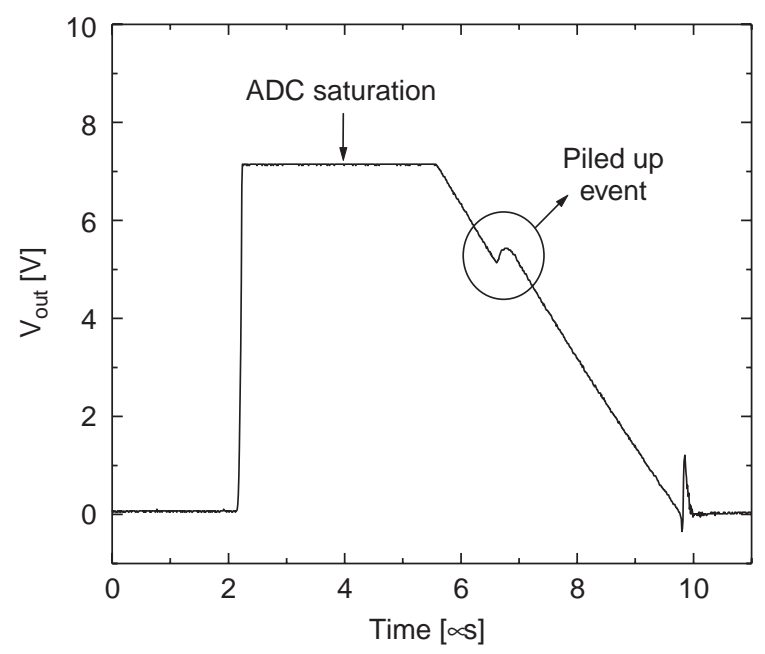

Fig. 10. Fast-reset transient with a piled-up low-energy event. It makes the reset time longer.

the $R_{0}$ is properly trimmed the exponential decay of $V_{\mathrm{IN}}$ is canceled out and the new pure exponential decay $\tau_{P}$ is introduced. Therefore, very simply

$V_{\mathrm{PZ1}}=(h+H) \mathrm{e}^{-\frac{t}{\tau_{P}}}$.

We calculate now $V_{\mathrm{PZ} 2}$. Assuming that $R_{0} \ll R_{1}$ and putting $V_{\mathrm{IN}}=0$, we easily find

$$
V_{\mathrm{PZ} 2}=-I R_{1} \oplus R_{2}\left(1-\mathrm{e}^{-\frac{t}{\tau_{P}}}\right) .
$$

Eventually

$$
\begin{aligned}
V_{\mathrm{PZ}}= & V_{\mathrm{PZ} 1}+V_{\mathrm{PZ} 2}=\left(h+H+I R_{1} \oplus R_{2}\right) \mathrm{e}^{-\frac{t}{\tau_{P}}} \\
& -I R_{1} \oplus R_{2} .
\end{aligned}
$$

\section{Acknowledgements}

The authors thank D. Bazzacco, R. Isocrate, G. Pascovici, C. Boiano and R. Bassini for their useful suggestions. We also wish to thank D. Bazzacco for hospitality in Padova.

\section{References}

[1] I. Kipnis, T. Collins, J. DeWitt, S. Dow, A. Frey, A. Grillo, et al., IEEE Trans. Nucl. Sci. NS-44 (3) (1997) 289.

[2] P.F. Manfredi, A. Leona, E. Mandelli, A. Perazzo, V. Re, Nucl. Instr. and Meth. A 439 (2-3) (2000) 361.

[3] D. Bazzacco, B. Cederwall, J. Cresswell, G. Duchene, J. Eberth, W. Gast, et al., AGATA Technical Proposal [online]. Available: http://agata.pd.infn.it/documents/Agata-proposal.pdf

[4] I.Y. Lee, Nucl. Instr. and Meth. A 422 (1999) 195.

[5] F. Camera, private communication. 